\title{
Association of Pioglitazone with Increased Risk of Prostate Cancer and Pancreatic Cancer: A Functional Network Study
}

\author{
Weiheng Wen · Peili Wu · Jinru Gong · Min Zhao · Zhen Zhang • \\ Rongping Chen · Hong Chen · Jia Sun (D)
}

Received: July 28, 2018 / Published online: September 25, 2018

(C) The Author(s) 2018

\begin{abstract}
Introduction: The question of whether pioglitazone, an antidiabetic drug, increases the risk of cancer has been debated for some time. Recent studies have shown that pioglitazone use can increase the risk of prostate cancer as well as pancreatic cancer. However, it is unclear whether pioglitazone is a causal risk factor for these cancers.

Methods: In this study, we aimed to explore the direct targets of pioglitazone and genes associated with this drug by querying open platforms in order to construct a biological function network, and then to further evaluate the
\end{abstract}

Weiheng Wen, Peili Wu and Jinru Gong contributed equally to this work.

Enhanced Digital Features To view enhanced digital features for this article go to https://doi.org/10.6084/ m9.figshare.7043330.

W. Wen $\cdot$ P. Wu $\cdot$ M. Zhao $\cdot$ Z. Zhang $\cdot$ R. Chen $\cdot$

H. Chen $(\bowtie) \cdot$ J. Sun $(\bowtie)$

Department of Endocrinology, Zhujiang Hospital, Southern Medical University, Guangzhou, People's Republic of China

e-mail: chenhong123@smu.edu.cn

J. Sun

e-mail: sunjia@smu.edu.cn

J. Gong

State Key Laboratory of Respiratory Disease, The

First Affiliated Hospital of Guangzhou Medical

University, Guangzhou, People's Republic of China relationships of pioglitazone with prostate cancer and pancreatic cancer.

Results: We first tested our hypothesis using DrugBank and STRING. We identified four direct targets of pioglitazone and 50 pioglitazone-associated genes, which were then selected for KEGG pathway analysis using STRING and WebGestalt. This analysis generated the top 25 KEGG pathways, among which four pathways were related to site-specific cancers, including prostate cancer and pancreatic cancer. Finally, a genomic study using cBioPortal indicated that genomic alterations of two gene sets related to the prostate cancer and pancreatic cancer pathways, respectively, are associated with the acceleration of carcinogenesis.

Conclusions: Pioglitazone is likely to be a causal risk factor for prostate cancer and pancreatic cancer, so this drug should be used with caution. The present research also demonstrates the use of biological function network analysis to effectively explore drug interactions and drug safety profiles.

Keywords: Bioinformatics; Cancer risk; Connectivity; Diabetic treatment; Drug safety; Functional network study; Pancreatic cancer; Pioglitazone; Prostate cancer; Side effect

\section{INTRODUCTION}

The global prevalence of diabetes and cancer is increasing rapidly. In 2015, an estimated 415 
million people were diagnosed with diabetes, and $90 \%$ of those were identified as having type 2 diabetes [1]. Recently, drugs used for the treatment of type 2 diabetes have been reported to affect the risk of cancer [2], suggesting that it is important to select appropriate hypoglycemic drugs that minimize the risk of cancer. Pioglitazone is a thiazolidinedione drug that regulates blood glucose homeostasis by binding to peroxisome proliferator-activated receptor gamma $(\operatorname{PPAR} \gamma)$. This drug can improve insulin resistance in peripheral tissues and the liver, protect B-cell function in diabetic patients, and is considered to be beneficial for lipid homeostasis [3]. Various restrictions have been placed on the utilization of other thiazolidinedione drugs, including troglitazone and rosiglitazone, due to the resulting risk of hepatotoxicity or cardiovascular disease [4]. Pioglitazone is currently the only thiazolidinedione drug that is commonly used globally.

However, the safety of pioglitazone still warrants investigation. It has been reported that the use of pioglitazone increases the incidence of bladder cancer [5-7]. However, the association between pioglitazone and the risk of bladder cancer remains a source of debate among researchers $[8,9]$. In addition to bladder cancer, researchers should also be aware that pioglitazone increases the risk of other types of cancers. In a study by Ferrara et al., pioglitazone use was found to be associated with an increased prostate cancer risk and pancreatic cancer risk, but it did not increase the risk of ten other cancers, including bladder cancer [10]. Also, a metaanalysis indicated that pioglitazone is associated with an increased incidence of prostate cancer and pancreatic cancer. Further investigation is needed to evaluate whether pioglitazone is a causal risk factor for these cancers.

In this research, we used DrugBank to obtain direct-target information for pioglitazone and investigate its potential pharmacological effects. Based on the four direct targets found for pioglitazone, 50 target-related genes were identified with the STRING database. We then performed pathway enrichment analysis for the targets and their associated genes using the STRING and WebGestalt databases. Interestingly, the results indicated that the prostate cancer pathway and the pancreatic cancer pathway were highly correlated with the functional network of pioglitazone. These pathways were thus screened, and the related genes were then used as queries in searches of the cBioPortal database to explore relevant genomic alterations. Overall, our research plan was to assess the risk of prostate cancer and pancreatic cancer associated with pioglitazone use via systematic network studies in order to determine the safety of pioglitazone in T2DM patients.

\section{METHODS}

\section{Drug-Target Search}

The DrugBank database is a useful web-based bioinformatics tool that can provide detailed drug data, including pharmacological mechanisms and targets $[11,12]$. In this study, DrugBank was used to retrieve information on pioglitazone, including the interactions between pioglitazone and its primary targets, in order to gain insight into the biological network of pioglitazone.

\section{Network Generation and Pathway Enrichment Analysis}

STRING v.10.5 is an open database that is used to explore protein-protein interactions and obtain information on interactions predicted by comparative genomics [13]. In the present research, the target proteins of pioglitazone were explored using STRING, and the results generated were then visualized using Cytoscape (version 3.6.0) [14]. In addition, KEGG pathway analysis of pioglitazoneassociated genes was conducted using STRING, which yielded the top 25 statistically significant KEGG enrichment pathways. WebGestalt is an integrated enrichment analysis tool that allows flexible and accurate exploration of functionally enriched pathways [15]. In our study, the pathway enrichment analysis of pioglitazone-associated genes was further validated using WebGestalt.

\section{Obtaining Cancer Genomics Data Linked to Pioglitazone Using cBioPortal}

cBioPortal is a web-based tool for exploring multidimensional cancer genomics data obtained from various cancer samples $[16,17]$. 
In our study, the cBioPortal database was used to explore the connectivity of pioglitazone-related genes across prostate cancer and pancreatic cancer studies.

\section{Compliance with Ethics Guidelines}

This article is based on previously conducted studies and does not contain any studies with human participants or animals performed by any of the authors.

\section{RESULTS}

\section{Characterization of the Biological Effects of Pioglitazone Using DrugBank and Visualization of the Pioglitazone Linkage Network Using STRING}

In this research, we first queried DrugBank using pioglitazone as an input, which yielded an output of DB01132, categorizing pioglitazone as a blood glucose lowering agent, cytochrome P450 enzyme inhibitor/inducer, peroxisome proliferator-activated receptor alpha/gamma agonist, and thiazolidinedione (Table 1). Among these, the primary use of pioglitazone is to improve glycemic control, where it is used as an adjunct to diet and exercise. Table 2 presents four primary targets of pioglitazone, PPARG, PPARD, PPARA, and MAOB. To further extend our research and analysis, STRING was employed to obtain 50 pioglitazone-related target proteins. These data, including the four primary targets, were then grouped together using Cytoscape 3.6 to build a biological function network for visualizing the interactions of the pioglitazone-related proteins: the four primary direct targets and the secondary pioglitazone-associated proteins. PPARA had 44 protein targets, PPARD had 19 protein targets, PPARG had 35 protein targets, and MAOB had 2 protein targets; see Fig. 1 . Three of the primary direct targets of pioglitazone (the exception being MAOB) had 17 genes in common.

\section{Analysis of Biologic Functions Related to Pioglitazone-Mediated Changes in Genes Using STRING and WebGestalt}

To assess the functional attributes of the pioglitazone-mediated genes, we performed KEGG pathway analysis using STRING. The top 25 KEGG enrichment pathways related to pioglitazone included the PPAR signaling pathway (16 genes), adipocytokine signaling pathway (12 genes), thyroid hormone signaling pathway (11 genes), cancer pathways (13 genes), AMPK signaling pathway (9 genes), prostate cancer (8 genes), hepatitis B (9 genes), non-alcoholic fatty liver disease ( 8 genes), T-cell receptor signaling pathway (7 genes), inflammatory bowel disease (6

Table 1 Characterization of pioglitazone using DrugBank

\begin{tabular}{|c|c|c|c|c|}
\hline DB_ID & Name & Group & Category & Indication \\
\hline \multirow[t]{4}{*}{ DB01132 } & \multirow[t]{4}{*}{ Pioglitazone } & \multirow{4}{*}{$\begin{array}{l}\text { Approved, } \\
\text { Investigational }\end{array}$} & Blood glucose lowering agents & \multirow{4}{*}{$\begin{array}{l}\text { As an adjunct to diet and exercise to } \\
\text { improve glycemic control in } \\
\text { adults with T2DM }\end{array}$} \\
\hline & & & $\begin{array}{l}\text { Cytochrome P450 enzyme inducers/ } \\
\text { inhibitors }\end{array}$ & \\
\hline & & & $\begin{array}{l}\text { Peroxisome proliferator-activated } \\
\text { receptor alpha/gamma agonists }\end{array}$ & \\
\hline & & & Thiazolidinediones & \\
\hline
\end{tabular}

Table 2 Identification of the direct targets of pioglitazone using DrugBank

\begin{tabular}{llllll}
\hline DB_ID & Name & Target & UniProt ID & Actions & Organism \\
\hline DB01132 & Pioglitazone & PPARG & P37231 & Agonist & Human \\
DB01132 & Pioglitazone & PPARD & Q03181 & Unknown & Human \\
DB01132 & Pioglitazone & PPARA & Q07869 & Unknown & Human \\
DB01132 & Pioglitazone & MAOB & P27338 & Unknown & Human \\
\hline
\end{tabular}




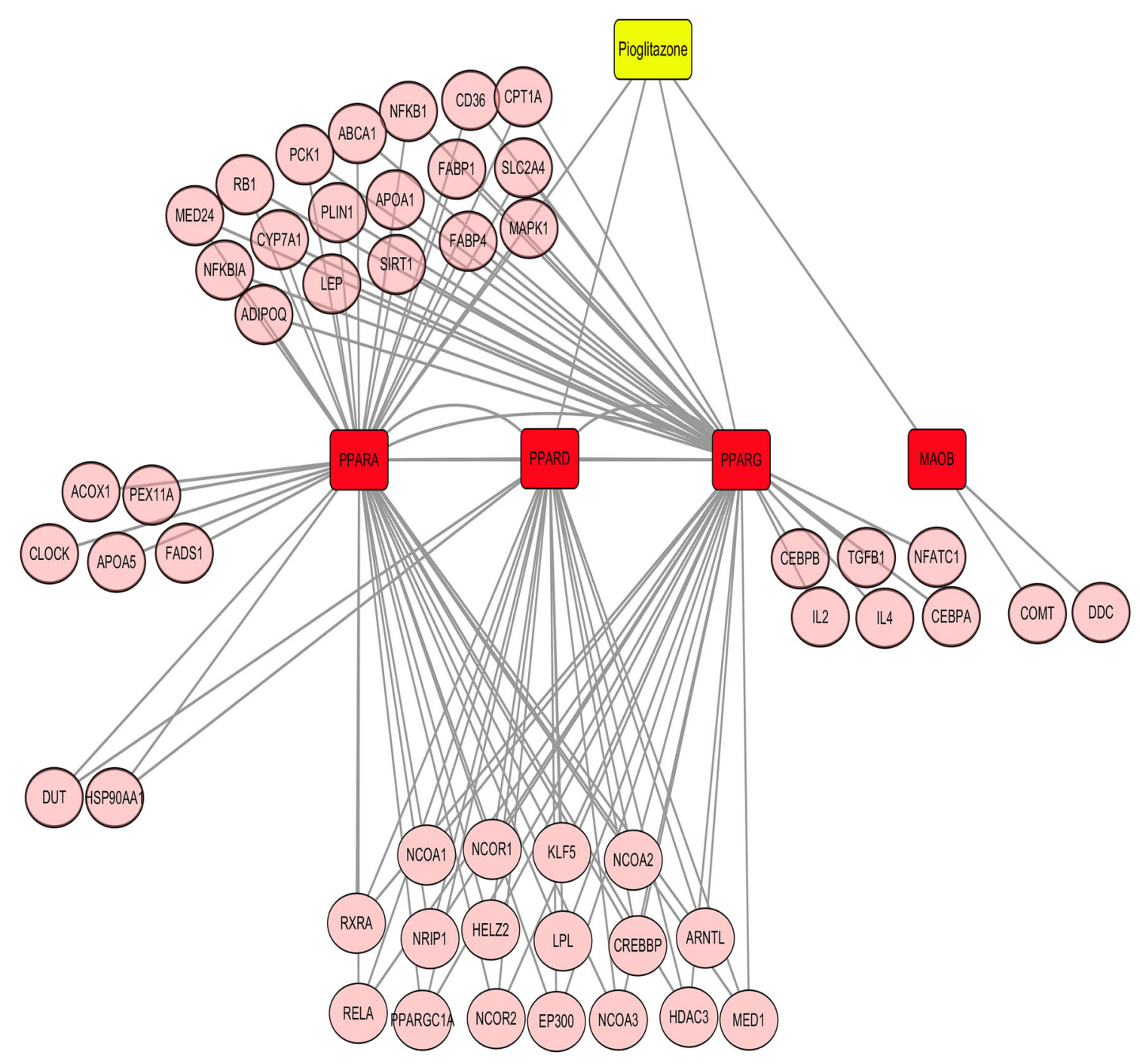

Fig. 1 Drug-target interactome of pioglitazone. The drug pioglitazone is highlighted in yellow, the primary direct protein targets (PPARA, PPARD, PPARG, and MAOB)

genes), HTLV-I infection (9 genes), FoxO signaling pathway (7 genes), leishmaniasis (6 genes), viral carcinogenesis (8 genes), chronic myeloid leukemia (6 genes), osteoclast differentiation (7 genes), Chagas disease (6 genes), transcriptional misregulation in cancer (7 genes), tuberculosis (7 genes), NOD-like receptor signaling pathway (5 genes), herpes simplex infection (7 genes), acute myeloid leukemia (5 genes), pancreatic cancer (5 genes), Epstein-Barr virus infection (7 genes), and B-cell receptor signaling pathway (5 genes) (Fig. 2). All identified enrichment pathways with biological functions were statistically significant, thus are highlighted in red, and the secondary pioglitazoneassociated proteins are highlighted in pink

motivating further study. As shown in Table 3, the most common enrichment pathway was the PPAR signaling pathway, which is consistent with pioglitazone mainly exerting a hypoglycemic effect. In addition, both the PPAR and adipocytokine signaling pathways are known to regulate glucose and lipid homeostasis, which is also characteristic of pioglitazone. These observations confirm the reliability of our results. Extensive grouping via biologic functional analysis also indicated that pioglitazone-associated genes are closely linked to cancer and its related signaling pathways, such as the FoxO signaling pathway 


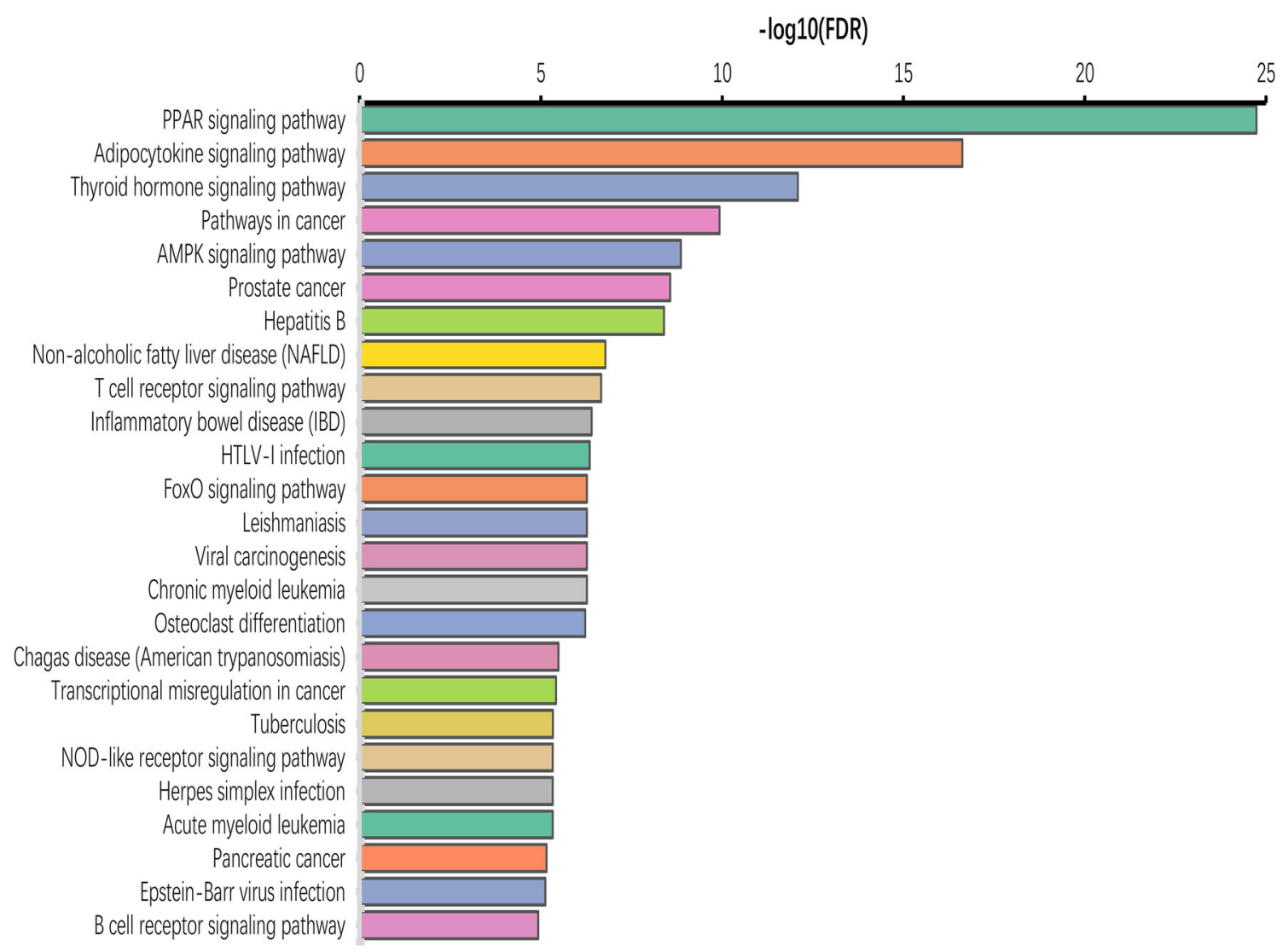

Fig. 2 KEGG pathway analysis of the potential functions of pioglitazone-associated genes in the pathway using STRING

[18], viral carcinogenesis [19], and transcriptional misregulation in cancer [20]. Among the 25 signaling enrichment pathways, four were directly linked to specific types of cancers: prostate cancer, chronic myeloid leukemia, acute myeloid leukemia, and pancreatic cancer. Notably, Ferrara et al. found that the use of pioglitazone can increase the risk of prostate cancer and pancreatic cancer but not ten other cancers (not including chronic/ acute myeloid leukemia). A meta-analysis also showed that pioglitazone use is associated with an increased risk of prostate cancer and pancreatic cancer [21]. These results are consistent with those from the pathway enrichment analysis of pioglitazone. However, whether the increased risk of prostate cancer and pancreatic cancer after treatment with pioglitazone is actually caused by the pioglitazone treatment was unclear. Therefore, the prostate cancer and pancreatic pathways were screened for further investigation.

Next, to verify the enrichment pathway identified by STRING, we additionally conducted
KEGG pathway enrichment using WebGestalt. The prostate cancer and pancreatic cancer pathways were also identified with statistical significance in this study (Table 4). A broad search indicated that eight genes in the prostate cancer pathway (CREBBP, EP300, HSP90AA1, NFKB1, NFKBIA, MAPK1, RB1, and RELA) and five genes in the pancreatic pathway (NFKB1, MAPK1, RB1, RELA, and TGFB1) were linked to pioglitazone. The CREBBP, EP300, and RELA genes in the prostate cancer pathway and the RELA gene in the pancreatic cancer pathway were all linked to three of the primary direct targets of pioglitazone.

\section{Mining Genomic Alterations Related to Pioglitazone-Associated Genes in Prostate Cancer Using cBioPortal}

To further investigate the associations between pioglitazone-associated genes and the prostate cancer pathway, cBioPortal was used to reveal 
Table 3 List of enriched pioglitazone-associated gene sets obtained using STRING

\begin{tabular}{|c|c|c|c|}
\hline Pathway description & \#Gene & Genes (corresponding gene set) & FDR \\
\hline PPAR signaling pathway & 16 & $\begin{array}{l}\text { ACOX1, ADIPOQ, APOA1, APOA5, CD36, CPT1A, CYP7A1, FABP1, } \\
\text { FABP4, LPL, PCK1, PLIN1, PPARA, PPARD, PPARG, RXRA }\end{array}$ & $1.82 \times 10^{-25}$ \\
\hline $\begin{array}{l}\text { Adipocytokine signaling } \\
\text { pathway }\end{array}$ & 12 & $\begin{array}{l}\text { ADIPOQ, CD36, CPT1A, LEP, NFKB1, NFKBIA, PCK1, PPARA, } \\
\text { PPARGC1A, RELA, RXRA, SLC2A4 }\end{array}$ & $2.38 \times 10^{-17}$ \\
\hline $\begin{array}{l}\text { Thyroid hormone } \\
\text { signaling pathway }\end{array}$ & 11 & $\begin{array}{l}\text { CREBBP, EP300, HDAC3, MAPK1, MED1, MED24, NCOA1, NCOA2, } \\
\text { NCOA3, NCOR1, RXRA }\end{array}$ & $8.12 \times 10^{-13}$ \\
\hline Pathways in cancer & 13 & $\begin{array}{l}\text { CEBPA, CREBBP, EP300, HSP90AA1, MAPK1, NFKB1, NFKBIA, PPARD, } \\
\text { PPARG, RB1, RELA, RXRA, TGFB1 }\end{array}$ & $1.18 \times 10^{-10}$ \\
\hline AMPK signaling pathway & 9 & ADIPOQ, CD36, CPT1A, LEP, PCK1, PPARG, PPARGC1A, SIRT1, SLC2A4 & $1.37 \times 10^{-9}$ \\
\hline Prostate cancer & 8 & CREBBP, EP300, HSP90AA1, MAPK1, NFKB1, NFKBIA, RB1, RELA & $2.70 \times 10^{-9}$ \\
\hline Hepatitis B & 9 & CREBBP, EP300, MAPK1, NFATC1, NFKB1, NFKBIA, RB1, RELA, TGFB1 & $4.00 \times 10^{-9}$ \\
\hline $\begin{array}{l}\text { Non-alcoholic fatty liver } \\
\text { disease (NAFLD) }\end{array}$ & 8 & ADIPOQ, CEBPA, LEP, NFKB1, PPARA, RELA, RXRA, TGFB1 & $1.66 \times 10^{-7}$ \\
\hline $\begin{array}{l}\text { T cell receptor signaling } \\
\text { pathway }\end{array}$ & 7 & IL2, IL4, MAPK1, NFATC1, NFKB1, NFKBIA, RELA & $2.19 \times 10^{-7}$ \\
\hline $\begin{array}{l}\text { Inflammatory bowel } \\
\text { disease (IBD) }\end{array}$ & 6 & IL2, IL4, NFATC1, NFKB1, RELA, TGFB1 & $4.00 \times 10^{-7}$ \\
\hline HTLV-I infection & 9 & CREBBP, EP300, IL2, NFATC1, NFKB1, NFKBIA, RB1, RELA, TGFB1 & $4.52 \times 10^{-7}$ \\
\hline FoxO signaling pathway & 7 & CREBBP, EP300, MAPK1, PCK1, SIRT1, SLC2A4, TGFB1 & $5.40 \times 10^{-7}$ \\
\hline Leishmaniasis & 6 & IL4, MAPK1, NFKB1, NFKBIA, RELA, TGFB1 & $5.40 \times 10^{-7}$ \\
\hline Viral carcinogenesis & 8 & CREBBP, EP300, HDAC3, MAPK1, NFKB1, NFKBIA, RB1, RELA & $5.40 \times 10^{-7}$ \\
\hline Chronic myeloid leukemia & 6 & MAPK1, NFKB1, NFKBIA, RB1, RELA, TGFB1 & $5.40 \times 10^{-7}$ \\
\hline Osteoclast differentiation & 7 & IL2, MAPK1, NFKB1, NFKBIA, RELA, TGFB1 & $6.01 \times 10^{-7}$ \\
\hline $\begin{array}{l}\text { Chagas disease (American } \\
\text { trypanosomiasis) }\end{array}$ & 6 & CEBPA, CEBPB, NCOR1, NFKB1, PPARG, RELA, RXRA & $3.32 \times 10^{-6}$ \\
\hline $\begin{array}{l}\text { Transcriptional } \\
\text { misregulation in cancer }\end{array}$ & 7 & CEBPB, CREBBP, EP300, MAPK1, NFKB1, RELA, TGFB1 & $3.85 \times 10^{-6}$ \\
\hline Tuberculosis & 7 & HSP90AA1, MAPK1, NFKB1, NFKBIA, RELA & $4.66 \times 10^{-6}$ \\
\hline $\begin{array}{l}\text { NOD-like receptor } \\
\text { signaling pathway }\end{array}$ & 5 & ARNTL, CLOCK, CREBBP, EP300, NFKB1, NFKBIA, RELA & $4.77 \times 10^{-6}$ \\
\hline Herpes simplex infection & 7 & CEBPA, MAPK1, NFKB1, PPARD, RELA & $4.77 \times 10^{-6}$ \\
\hline Acute myeloid leukemia & 5 & MAPK1, NFKB1, RB1, RELA, TGFB1 & $4.77 \times 10^{-6}$ \\
\hline Pancreatic cancer & 5 & CREBBP, EP300, NCOR2, NFKB1, NFKBIA, RB1, RELA & $7.03 \times 10^{-6}$ \\
\hline $\begin{array}{l}\text { Epstein-Barr virus } \\
\text { infection }\end{array}$ & 7 & MAPK1, NFATC1, NFKB1, NFKBIA, RELA & $7.54 \times 10^{-6}$ \\
\hline $\begin{array}{l}\text { B-cell receptor signaling } \\
\text { pathway }\end{array}$ & 5 & IL2, MAPK1, NFKB1, NFKBIA, RELA, TGFB1 & $1.19 \times 10^{-5}$ \\
\hline
\end{tabular}

genomic alterations of pioglitazone-associated genes in prostate cancer. A total of 14 prostate cancer studies were included in cBioPortal. One study was only accepted provisionally, so we focused on the remaining 13 studies. Gene sets containing eight identified genes (EGF, EGFR, THBS1, VEGFA) associated with prostate cancer were analyzed in the 13 studies. The results 
Table 4 List of enriched pioglitazone-related gene sets obtained via WebGestalt

\begin{tabular}{|c|c|c|c|}
\hline Pathway name & \#Gene & Genes (corresponding gene set) & Statistics \\
\hline $\begin{array}{l}\text { PPAR signaling } \\
\text { pathway }\end{array}$ & 16 & $\begin{array}{l}\text { APOA5, CPT1A, CYP7A1, FABP4, FABP1, APOA1, LPL, } \\
\text { ACOX1, PCK1, PLIN1, PPARA, PPARD, PPARG, RXRA, } \\
\text { ADIPOQ, CD36 }\end{array}$ & $\begin{array}{l}C=72, O=16, E=0.51 \\
R=31.26, P=0\end{array}$ \\
\hline $\begin{array}{l}\text { Adipocytokine } \\
\text { signaling pathway }\end{array}$ & 12 & $\begin{array}{l}\text { PPARGC1A, CPT1A, LEP, NFKB1, NFKBIA, PCK1, PPARA, } \\
\text { RELA, RXRA, SLC2A4, ADIPOQ, CD36 }\end{array}$ & $\begin{array}{l}C=70, O=12, E=0.5 \\
R=24.12, P=3.26 \times 10^{-14}\end{array}$ \\
\hline $\begin{array}{l}\text { Thyroid hormone } \\
\text { signaling pathway }\end{array}$ & 11 & $\begin{array}{l}\text { NCOA2, CREBBP, EP300, MED1, MAPK1, RXRA, NCOA3, } \\
\text { NCOA1, HDAC3, NCOR1, MED24 }\end{array}$ & $\begin{array}{l}C=118, O=11, E=0.84 \\
R=13.11, P=4.07 \times 10^{-10}\end{array}$ \\
\hline $\begin{array}{l}\text { Th17 cell } \\
\text { differentiation }\end{array}$ & 10 & $\begin{array}{l}\text { HSP90AA1, IL2, IL4, NFATC1, NFKB1, NFKBIA, MAPK1, } \\
\text { RELA, RXRA, TGFB1 }\end{array}$ & $\begin{array}{l}C=107, O=10, E=0.76 \\
R=13.15, P=2.73 \times 10^{-9}\end{array}$ \\
\hline Insulin resistance & 9 & $\begin{array}{l}\text { PPARGC1A, CPT1A, NFKB1, NFKBIA, PCK1, PPARA, RELA, } \\
\text { SLC2A4, CD36 }\end{array}$ & $\begin{array}{l}C=109, O=9, E=0.77 \\
R=11.62, P=5.52 \times 10^{-8}\end{array}$ \\
\hline Prostate cancer & 8 & $\begin{array}{l}\text { CREBBP, EP300, HSP90AA1, NFKB1, NFKBIA, MAPK1, RB1, } \\
\text { RELA }\end{array}$ & $\begin{array}{l}C=89, O=8, E=0.63 \\
R=12.65, P=1.68 \times 10^{-7}\end{array}$ \\
\hline $\begin{array}{l}\text { AMPK signaling } \\
\text { pathway }\end{array}$ & 9 & $\begin{array}{l}\text { PPARGC1A, CPT1A, SIRT1, LEP, PCK1, PPARG, SLC2A4, } \\
\text { ADIPOQ, CD36 }\end{array}$ & $\begin{array}{l}C=124, O=9, E=0.88 \\
R=10.21, P=1.70 \times 10^{-7}\end{array}$ \\
\hline Hepatitis B & 9 & $\begin{array}{l}\text { CREBBP, EP300, NFATC1, NFKB1, NFKBIA, MAPK1, RB1, } \\
\text { RELA, TGFB1 }\end{array}$ & $\begin{array}{l}C=146, O=9, E=1.04 \\
R=8.672, P=6.87 \times 10^{-7}\end{array}$ \\
\hline Pathways in cancer & 13 & $\begin{array}{l}\text { CEBPA, CREBBP, EP300, HSP90AA1, NFKB1, NFKBIA, PPARD, } \\
\text { PPARG, MAPK1, RB1, RELA, RXRA, TGFB1 }\end{array}$ & $\begin{array}{l}C=397, O=13, E=2.82 \\
R=4.607, P=2.60 \times 10^{-6}\end{array}$ \\
\hline $\begin{array}{l}\text { Th1 and Th2 cell } \\
\text { differentiation }\end{array}$ & 7 & IL2, IL4, NFATC1, NFKB1, NFKBIA, MAPK1, RELA & $\begin{array}{l}C=92, O=7, E=0.65 \\
R=10.7, P=3.31 \times 10^{-6}\end{array}$ \\
\hline $\begin{array}{l}\text { Inflammatory bowel } \\
\text { disease (IBD) }\end{array}$ & 6 & IL2, IL4, NFATC1, NFKB1, RELA, TGFB1 & $\begin{array}{l}C=65, O=6, E=0.46 \\
R=12.99, P=5.72 \times 10^{-6}\end{array}$ \\
\hline $\begin{array}{l}\text { Glucagon signaling } \\
\text { pathway }\end{array}$ & 7 & PPARGC1A, CPT1A, CREBBP, EP300, SIRT1, PCK1, PPARA & $\begin{array}{l}C=103, O=7, E=0.73 \\
R=9.561, P=7.06 \times 10^{-6}\end{array}$ \\
\hline $\begin{array}{l}\text { T-cell receptor } \\
\text { signaling pathway }\end{array}$ & 7 & IL2, IL4, NFATC1, NFKB1, NFKBIA, MAPK1, RELA & $\begin{array}{l}C=105, O=7, E=0.75 \\
R=9.379, P=8.02 \times 10^{-6}\end{array}$ \\
\hline $\begin{array}{l}\text { cAMP signaling } \\
\text { pathway }\end{array}$ & 9 & $\begin{array}{l}\text { CREBBP, EP300, NFATC1, NFKB1, NFKBIA, ACOX1, PPARA, } \\
\text { MAPK1, RELA }\end{array}$ & $\begin{array}{l}C=200, O=9, E=1.42 \\
R=6.331, P=9.36 \times 10^{-6}\end{array}$ \\
\hline $\begin{array}{l}\text { Non-alcoholic fatty } \\
\text { liver disease } \\
\text { (NAFLD) }\end{array}$ & 8 & CEBPA, LEP, NFKB1, PPARA, RELA, RXRA, TGFB1, ADIPOQ & $\begin{array}{l}C=151, O=8, E=1.07 \\
R=7.454, P=9.45 \times 10^{-6}\end{array}$ \\
\hline Leishmaniasis & 6 & IL4, NFKB1, NFKBIA, MAPK1, RELA, TGFB1 & $\begin{array}{l}C=73, O=6, E=0.52 \\
R=11.56, P=1.13 \times 10^{-5}\end{array}$ \\
\hline $\begin{array}{l}\text { Chronic myeloid } \\
\text { leukemia }\end{array}$ & 6 & NFKB1, NFKBIA, MAPK1, RB1, RELA, TGFB1 & $\begin{array}{l}C=73, O=6, E=0.52 \\
R=11.56, P=1.13 \times 10^{-5}\end{array}$ \\
\hline $\begin{array}{l}\text { Osteoclast } \\
\text { differentiation }\end{array}$ & 7 & NFATC1, NFKB1, NFKBIA, PPARG, MAPK1, RELA, TGFB1 & $\begin{array}{l}C=132, O=7, E=0.94 \\
R=7.461, P=3.59 \times 10^{-5}\end{array}$ \\
\hline
\end{tabular}


Table 4 continued

\begin{tabular}{|c|c|c|c|}
\hline Pathway name & \#Gene & Genes (corresponding gene set) & Statistics \\
\hline $\begin{array}{l}\text { FoxO signaling } \\
\text { pathway }\end{array}$ & 7 & CREBBP, EP300, SIRT1, PCK1, MAPK1, SLC2A4, TGFB1 & $\begin{array}{l}C=134, O=7, E=0.95 \\
R=7.349, P=3.95 \times 10^{-5}\end{array}$ \\
\hline $\begin{array}{l}\text { Acute myeloid } \\
\text { leukemia }\end{array}$ & 5 & CEBPA, NFKB1, PPARD, MAPK1, RELA & $\begin{array}{l}C=57, O=5, E=0.41 \\
R=12.34, P=4.70 \times 10^{-5}\end{array}$ \\
\hline $\begin{array}{l}\text { Longevity-regulating } \\
\text { pathway }\end{array}$ & 6 & PPARGC1A, SIRT1, NFKB1, PPARG, RELA, ADIPOQ & $\begin{array}{l}C=94, O=6, E=0.67 \\
R=8.98, P=4.82 \times 10^{-5}\end{array}$ \\
\hline HTLV-I infection & 9 & $\begin{array}{l}\text { CREBBP, EP300, IL2, NFATC1, NFKB1, NFKBIA, RB1, RELA, } \\
\text { TGFB1 }\end{array}$ & $\begin{array}{l}C=258, O=9, E=1.83 \\
R=4.908, P=7.07 \times 10^{-5}\end{array}$ \\
\hline $\begin{array}{l}\text { Chagas disease } \\
\text { (American } \\
\text { trypanosomiasis) }\end{array}$ & 6 & IL2, NFKB1, NFKBIA, MAPK1, RELA, TGFB1 & $\begin{array}{l}C=104, O=6, E=0.74 \\
R=8.117, P=8.52 \times 10^{-5}\end{array}$ \\
\hline Viral carcinogenesis & 8 & $\begin{array}{l}\text { CREBBP, EP300, NFKB1, NFKBIA, MAPK1, RB1, RELA, } \\
\text { HDAC3 }\end{array}$ & $\begin{array}{l}C=205, O=8, E=1.46 \\
R=5.49, P=8.57 \times 10^{-5}\end{array}$ \\
\hline Pancreatic cancer & 5 & NFKB1, MAPK1, RB1, RELA, TGFB1 & $\begin{array}{l}C=65, O=5, E=0.47 \\
R=10.66, P=9.57 \times 10^{-5}\end{array}$ \\
\hline
\end{tabular}

$C$ number of genes referenced in the category, $O$ number of genes that overlap in gene set as well as category, $E$ expected number in the category, $R$ enrichment ratio, $P$ the $p$ value calculated via the hypergeometric test

showed that the most pronounced mutations detected in the identified gene sets occurred in Demichelis' study, with $46.73 \%$ genetic alterations [22]. In that study, 50 cases (46.73\%) had alterations in all eight genes; the alteration frequencies of the eight identified genes are shown in Fig. 3. For RELA (31\%), CREBBP (25\%), HSP90AA1 (25\%), NFKBIA (21\%), NFKB1 (12\%), MAPK (10\%), and EP300 (8\%), the most common alterations were amplifications. For RB1 $(12 \%)$, the majority of the alterations were deep deletions, with a small fraction of amplifications as well as missense mutations.

cBioPortal can also be used to perform interactive analysis and to construct networks that are altered in prostate cancer. Figure 4 shows a network including all neighbors of the eight identified genes CREBBP, EP300, HSP90AA1, NFKB1, NFKBIA, MAPK1, RB1, and RELA. To reduce the complexity of the analysis, we employed the alteration frequency within a particular prostate cancer study as a filter, so that only the genes that displayed high alteration frequencies were presented. The eight selected genes were found to be linked to AR using 49.5\% alteration as a filter. Eleven genes, including MYC and NCOA2, were flagged up using $44.8 \%$ alteration as a filter. Thirteen gene clusters, including AR, MYC, NCOA2, HEY1, and LY96, were highlighted by applying $43.9 \%$ alteration as a filter. This integrated network revealed the variability in the pioglitazoneassociated gene alterations among the prostate cancer samples in Demichelis' study [22].

\section{Mining Genomic Alterations Related to Pioglitazone-Associated Genes in Pancreatic Cancer Using cBioPortal}

cBioPortal was also used to assess genomic alterations of pioglitazone-associated genes in pancreatic cancer. A total of four pancreatic cancer studies were included in cBioPortal. One study was only accepted provisionally, so the remaining three studies were used to accurately evaluate the genomic alterations of five selected genes: RB1, MAPK1, NFKB1, RELA, and TGFB1. As shown in Fig. 5, alterations ranging from 1.01 to $29.36 \%$ were detected for the selected 


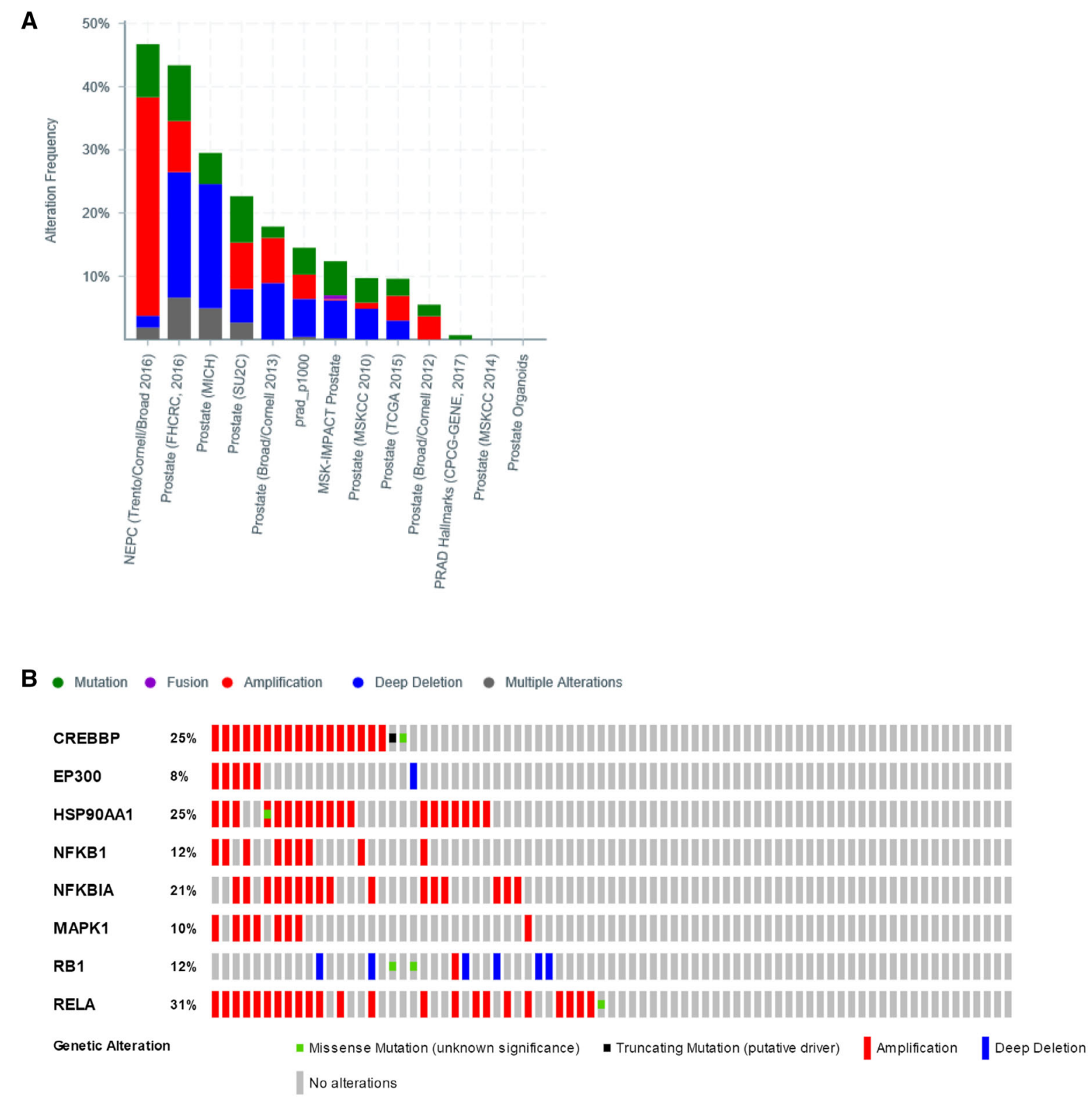

Fig. 3a-b Exploring the genetic alterations linked to the pioglitazone-related genes CREBBP, EP300, HSP90AA1, NFKB1, NFKBIA, MAPK1, RB1, and RELA in prostate cancer using cBioPortal. a Overview of variations in CREBBP, EP300, HSP90AA1, NFKB1, NFKBIA, MAPK1, $\mathrm{RB} 1$, and RELA in the genomic database across a series of

genes. The study by Knudsen [23] displayed the most pronounced genomic alterations. In that study, 32 cases showed alterations in all five genes. TGFB1 (16\%) showed the most prominent alterations, which included amplification with a few cases of missense mutation. For RELA prostate cancer samples. b Oncopoint: a visual display of genomic alterations in the eight identified genes (based on the Demichelis study [22]). Genomic alterations are summarized and displayed as \% changes in the selected genes. Each row corresponds to a gene, and each column is a sample. Bars with different colors represent different genomic alterations

$(10 \%)$, the majority of the alterations were amplifications. For MAPK1 (7\%), most of the alterations were amplifications, with a small fraction of deep deletions, missense mutations, and truncating mutations. For RB1 and NFKB1, only a few alterations were detected (5\% and 


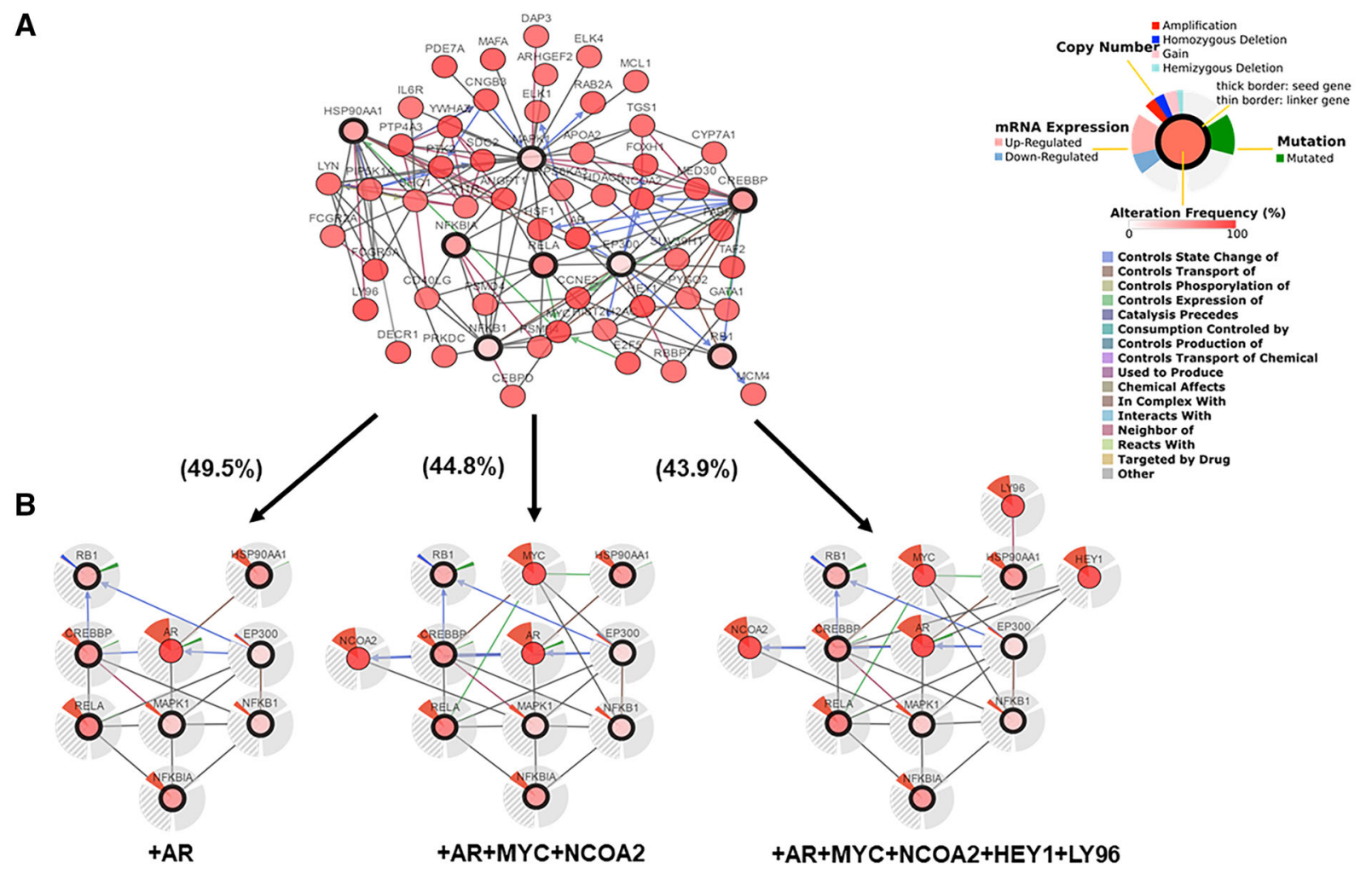

Fig. 4a-b A visual display of gene networks linked to CREBBP/EP300/HSP90AA1/NFKB1/NFKBIA/MAP

$\mathrm{K} 1 / \mathrm{RB} 1 / \mathrm{RELA}$ in prostate cancer (based on the Demichelis study [22]). a Eight selected pioglitazone-related genes were employed as seed genes (circles with thick black outlines) to harvest all the other genes that were changed in prostate

$2.8 \%$, respectively). A network study was then conducted which included neighbors of the five selected genes (Fig. 6). Six genes, including TP53, were identified using $54.1 \%$ alterations as the filter. Seven gene clusters, including TP53 and SMAD4, were obtained with a filter of $43.1 \%$ alterations, while eight genes, including TP53, SMAD4, and CDKN2A, were highlighted by a filter of $41.2 \%$ alterations.

\section{DISCUSSION}

Pioglitazone, a thiazolidinedione drug that regulates glucose homeostasis, has been widely used to treat diabetes since 1999 [24]. To our knowledge, the safety profile of pioglitazone has not been determined, including its affect on cancer risk. A cohort and case-control study showed

cancer samples using cBioPortal. b Neighboring genes associated with the eight selected genes were filtered by alteration (\%). Darker red indicates an increased frequency of alterations in prostate cancer. In addition to the selected genes, the filter used also involved the highest genomic alteration frequency in the prostate cancer study

that pioglitazone use is statistically significantly associated with an increased risk of prostate cancer and pancreatic cancer [10]. Meanwhile, a meta-analysis also reported that pioglitazone treatment may increase the risk of prostate cancer and pancreatic cancer [21]. Thus, it is necessary to investigate whether pioglitazone is a causal risk factor for these cancers.

In this study, we first constructed a biological network for pioglitazone to evaluate the relationship between pioglitazone and each type of cancer using open platforms. Four primary targets (PPARG, PPARD, PPARA, and MAOB) and another 50 pioglitazone-related proteins were identified using DrugBank and STRING, and then a KEGG pathway enrichment analysis was carried out based on the network for pioglitazone. As previously described, the relationships between pioglitazone use and prostate cancer 
A
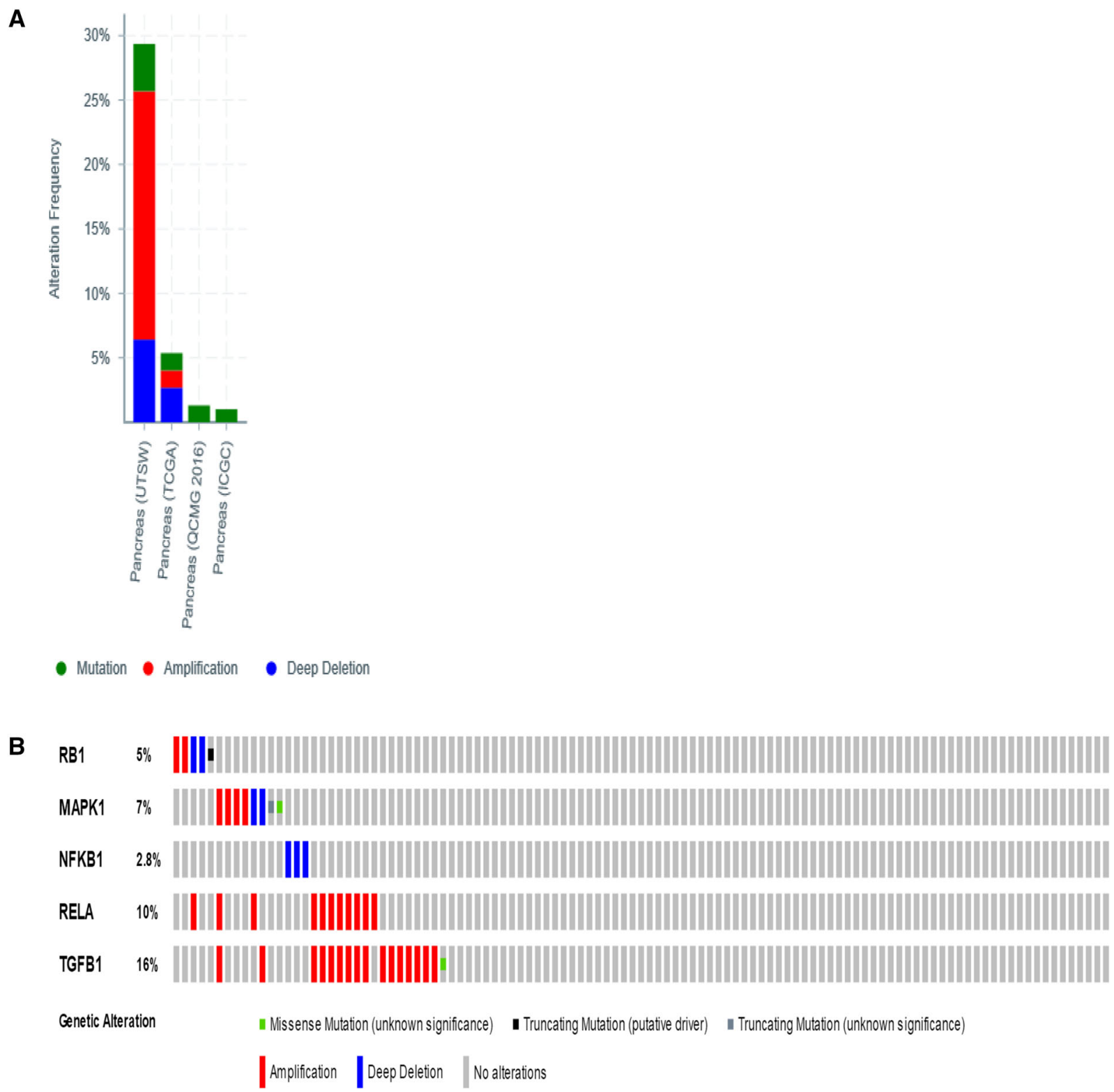

Fig. 5a-b Exploring genetic alterations linked to the pioglitazone-related genes RB1, MAPK1, NFKB1, RELA, and TGFB1 in pancreatic cancer using cBioPortal. a Overview of variations in RB1, MAPK1, NFKB1,

risk as well as pancreatic cancer risk remain unclear. Our KEGG enrichment analysis showed that among the top 25 enrichment pathways, four were directly linked to specific types of cancers: prostate cancer, chronic myeloid leukemia, acute myeloid leukemia, and pancreatic cancer. In their study, Ferrara et al. discovered that pioglitazone use can increase the risk of
RELA, and TGFB1 in the genomic database across a series of pancreatic cancer samples. b Oncopoint: a visual display of genomic alterations in the five identified genes (based on the Knudsen study [23])

prostate cancer and that of pancreatic cancer, but not the risk of ten other cancers (not including chronic/acute myeloid leukemia) [10]. Hongting et al. also found that the use of pioglitazone leads to an increased risk of prostate cancer as well as an increased risk of pancreatic cancer [21]. Therefore, the prostate cancer and pancreatic cancer pathways were 


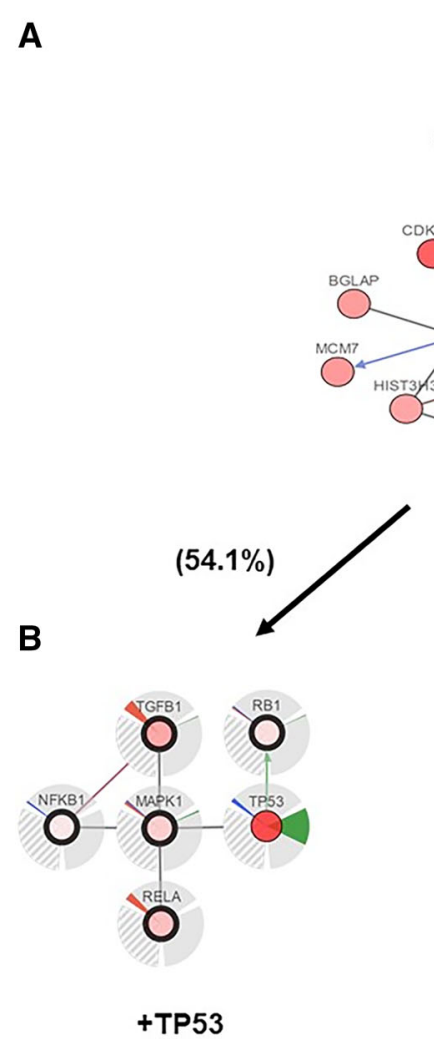

Fig. 6a-b A visual display of gene networks linked to $\mathrm{RB1}$ /MAPK1/NFKB1/RELA/TGFB1 in pancreatic cancer (based on the Knudsen study [23]). a Five selected pioglitazone-related genes to explore all the other genes

screened to determine whether pioglitazone is a causal risk factor for these cancers. For the prostate cancer pathway, eight identified genes (CREBBP, EP300, HSP90AA1, NFKB1, NFKBIA, MAPK1, RB1, and RELA) were selected, and then their genomic alterations were explored across prostate cancer studies using cBioPortal. Notably, the overlapping genes EP300, CREBBP, and RELA were all found to be linked to three of the primary direct targets of pioglitazone. Additionally, the results showed that the majority of the alterations to EP300, CREBBP, and RELA in prostate cancer were amplifications. These amplifications trigger an upregulation of the expression of those genes, which is associated with the acceleration of carcinogenesis $[25,26]$. Moreover, mutual exclusivity analysis showed that there were co-occurrence relationships between EP300 and CREBBP, EP300 and RELA, and CREBBP and RELA (data not shown),

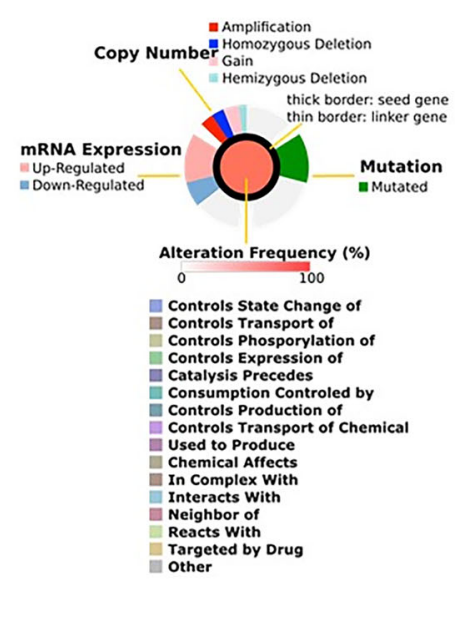

$(41.2 \%)$
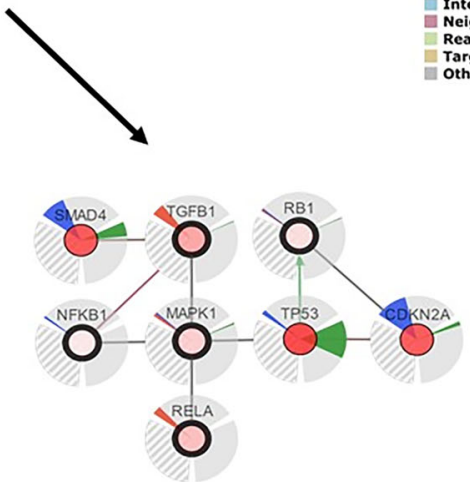

+TP53+SMAD4+CDKN2A

that were changed in pancreatic cancer samples using cBioPortal. b Neighboring genes associated with the five selected genes were filtered by alteration (\%)

demonstrating the synergistic effects of these genes in prostate cancer development.

For the pancreatic cancer pathway, five overlapping genes (NFKB1, MAPK1, RB1, RELA, and TGFB1) were extracted for further analysis. RELA was found to be associated with three of the primary direct targets of pioglitazone, and was observed to be amplified in pancreatic cancer. This results in the overexpression of this gene, which is linked to the carcinogenesis of pancreatic cancer [27]. The TGFB1 gene presented the most pronounced alterations, which were mainly amplifications, thus promoting the expression of TGFB1 and accounting for the development of pancreatic cancer [28]. A cooccurrence relationship between RELA and TGFB1 was noted (data not shown). Based on alteration analysis of the genes related to pioglitazone, it is clear that EP300, CREBBP, and RELA are strongly amplified in prostate cancer 
and that RELA and TGFB1 are strongly amplified in pancreatic cancer, suggesting that the increased risk of cancer development associated with pioglitazone is due to the amplification of these pioglitazone-related genes. Therefore, the results of our network analysis correlate well with the increased prostate cancer risk and pancreatic cancer risk associated with pioglitazone use.

Further study is still needed to explore the impact of pioglitazone on cancer. The expression profiles of PPAR $\gamma$ in tumor tissues are different from those in normal tissues, and PPAR $\gamma$ agonists have been reported to suppress tumor cell growth $[29,30]$. However, conflicting results regarding the pro-carcinogenic effects of PPAR $\gamma$ agonists such as pioglitazone can be found in several clinical studies $[8,24]$. Therefore, whether pioglitazone affects cancer development via the PPAR $\gamma$ signaling pathway remains unclear. Our KEGG pathway analysis showed that the AMPK, FoxO, and viral carcinogenesis pathways are closely associated with pioglitazone. These pathways are involved in the development of cancer $[18,19,31]$, so future research into the pioglitazone-induced carcinogenesis should also focus on those signaling pathways. In addition, there are various confounding factors in clinical studies, such as age, gender, race, lifestyle habits, occupational exposure, drug dose, the level of blood glucose control, and complications, which could contribute to the observed inconsistency of study results. The biological function network of pioglitazone that we constructed can eliminate these confounding factors, and the generated results may be close to the actual effects of pioglitazone on human beings. Our results also show that pioglitazone has the potential to affect chronic/acute myeloid leukemia-an association that has not received enough attention from researchers. Thus, constructing a gene functional network may be a useful supplemental method for evaluating the safety profiles of drugs.

It should be noted that hyperglycemia or insulin resistance also enhances the risk of malignancy [32], so using pioglitazone as an antidiabetic drug does have beneficial effects in this context too. However, pioglitazone may have other biological actions that contribute to the development of different types of cancers. The combination of these effects of pioglitazone may determine its impact on the risk of specific types of cancers. Therefore, when choosing an antidiabetic drug, priority should be given to drugs that can correct hyperglycemia without increasing the risk of cancer, assuming that all of the drugs considered permit similar control of glucose homeostasis.

There are several limitations of this study. First, although we comprehensively analyzed the observed effects of pioglitazone and its associations with proteins, this study lacks a functional validation experiment which directly demonstrates that pioglitazone does indeed increase the risk of prostate cancer and pancreatic cancer. Second, the results of the pathway enrichment analysis for specific types of cancer were obtained with the STRING database, but the interaction of pioglitazone-related targets does not imply the activation of partners; such interactions often have a neutral or sometimes negative impact on the partner's function. The nature of the interactions require further investigation to clarify the molecular mechanism through which pioglitazone increases the risk of prostate cancer and pancreatic cancer. Finally, other cancer pathways revealed by pathway enrichment analysis have not yet been investigated. Whether the functional network between pioglitazone and prostate/pancreatic cancer presented in this study can be extended to other specific types of cancers remains to be explored. However, the results of this study may provide the foundations for further experimental research and help researchers to translate basic research into clinical applications.

In summary, we integrated and utilized open databases to establish a biological effect network for pioglitazone and thereby explore the safety of this drug. Scientific research should lead to the discovery of more pioglitazone-targeted proteins helps update the functional network constructed, andthereby provide novel insights into scientific research, and permit improved clinical guidance. For example, our results suggest that pioglitazone is likely to be a causal risk factor for prostate cancer and pancreatic cancer. Therefore, government regulators, doctors, and patients should evaluate the 
risk-benefit relationship of pioglitazone when choosing a suitable hypoglycemic drug. Overall, this study has demonstrated a simple and flexible method for applying information about the biological network of a drug and genomic alterations during cancer to the rational analysis of drug safety.

\section{CONCLUSIONS}

Our results suggest that pioglitazone is likely to be a causal risk factor for both prostate cancer and pancreatic cancer.

\section{ACKNOWLEDGEMENTS}

Funding. This work and article processing charges for this publication were supported by the National Natural Science Foundation of China (grant numbers 81670783, 81570716, and 81770835); the Natural Science Foundation of Guangdong Province (grant numbers 2017A030313473, 2016A030313633]; and the National Undergraduate Training Programs for Innovation and Entrepreneurship (grant number 201712121049).

Authorship. All named authors meet the International Committee of Medical Journal Editors (ICMJE) criteria for authorship of this article, take responsibility for the integrity of the work as a whole, and have given their approval for this version to be published.

Disclosures. Weiheng Wen, Peili Wu, Jinru Gong, Min Zhao, Zhen Zhang, and Rongping Chen have nothing to declare. Hong Chen was supported by the National Natural Science Foundation of China (grant numbers 81570716 and 81770835) and the Natural Science Foundation of Guangdong Province (grant number 2016A030313633). Jia Sun has received funding from the National Natural Science Foundation of China (grant number 81670783), the Natural Science Foundation of Guangdong Province (grant number 2017A030313473) and the National Undergraduate Training Programs for
Innovation and Entrepreneurship (grant number 201712121049).

Compliance with Ethics Guidelines. This article is based on previously conducted studies and does not contain any studies with human participants or animals performed by any of the authors.

Data Availability. The research data used to prepare the manuscript are available from Dr. Sun Jia or Dr. Chen Hong on reasonable request.

Open Access. This article is distributed under the terms of the Creative Commons Attribution-NonCommercial 4.0 International License (http://creativecommons.org/licenses/ by-nc/4.0/), which permits any noncommercial use, distribution, and reproduction in any medium, provided you give appropriate credit to the original author(s) and the source, provide a link to the Creative Commons license, and indicate if changes were made.

\section{REFERENCES}

1. Ogurtsova K, da Rocha Fernandes JD, Huang Y, et al. IDF Diabetes Atlas: global estimates for the prevalence of diabetes for 2015 and 2040. Diabetes Res Clin Pract. 2017;128:40-50.

2. Tang GH, Satkunam M, Pond GR, et al. Association of metformin with breast cancer incidence and mortality in patients with type 2 diabetes: a GRADE assessed systematic review and meta-analysis. Cancer Epidemiol Biomark Prev. 2018;27(6):627-635.

3. Krishnaswami A, Ravi-Kumar S, Lewis JM. Thiazolidinediones: a 2010 perspective. Perm J. 2010;14(3):64-72.

4. Della-Morte D, Palmirotta R, Rehni AK, et al. Pharmacogenomics and pharmacogenetics of thiazolidinediones: role in diabetes and cardiovascular risk factors. Pharmacogenomics. 2014;15(16):2063-82.

5. Oleksiewicz MB, Southgate J, Iversen L, Egerod FL. Rat urinary bladder carcinogenesis by dual-acting PPARalpha + gamma agonists. PPAR Res. 2008;2008:103167.

6. Dormandy JA, Charbonnel B, Eckland DJ, et al. Secondary prevention of macrovascular events in 
patients with type 2 diabetes in the PROactive Study (PROspective pioglitAzone Clinical Trial In macroVascular Events): a randomised controlled trial. Lancet. 2005;366(9493):1279-89.

7. Scheen AJ. Outcomes and lessons from the PROactive study. Diabetes Res Clin Pract. 2012;98(2):175-86.

8. Erdmann E, Song E, Spanheimer R, van Troostenburg de Bruyn AR, Perez A. Observational follow-up of the PROactive study: a 6-year update. Diabetes Obes Metab. 2014;16(1):63-74.

9. Filipova E, Uzunova K, Kalinov K, Vekov T. Pioglitazone and the risk of bladder cancer: a meta-analysis. Diabetes Ther. 2017;8(4):705-26.

10. Lewis JD, Habel LA, Quesenberry CP, et al. Pioglitazone use and risk of bladder cancer and other common cancers in persons with diabetes. JAMA. 2015;314(3):265-77.

11. Wishart DS, Feunang YD, Guo AC, et al. DrugBank 5.0: a major update to the DrugBank database for 2018. Nucleic Acids Res. 2018;46(D1):D1074-d82.

12. Wishart DS, Knox C, Guo AC, et al. DrugBank: a comprehensive resource for in silico drug discovery and exploration. Nucleic Acids Res. 2006;34:D668-72.

13. Szklarczyk D, Morris JH, Cook H, et al. The STRING database in 2017: quality-controlled protein-protein association networks, made broadly accessible. Nucleic Acids Res. 2017;45(D1):D362-8.

14. Shannon P, Markiel A, Ozier O, et al. Cytoscape: a software environment for integrated models of biomolecular interaction networks. Genome Res. 2003;13(11):2498-504.

15. Wang J, Vasaikar S, Shi Z, Greer M, Zhang B. WebGestalt 2017: a more comprehensive, powerful, flexible and interactive gene set enrichment analysis toolkit. Nucleic Acids Res. 2017;45(W1):W130-7.

16. Gao J, Aksoy BA, Dogrusoz U, et al. Integrative analysis of complex cancer genomics and clinical profiles using the cBioPortal. Sci Signal. 2013;6(269):pl1.

17. Cerami E, Gao J, Dogrusoz U, et al. The cBio cancer genomics portal: an open platform for exploring multidimensional cancer genomics data. Cancer Discov. 2012;2(5):401-4.

18. Ma J, Matkar S, He X, Hua X. FOXO family in regulating cancer and metabolism. Semin Cancer Biol. 2018;50:32-41.

19. Smith AJ, Smith LA. Viral carcinogenesis. Prog Mol Biol Transl Sci. 2016;144:121-68.
20. Grimmer MR, Farnham PJ. Can genome engineering be used to target cancer-associated enhancers? Epigenomics. 2014;6(5):493-501.

21. Qu H, Zheng Y, Wang Y, et al. Global and regional effects of bladder cancer risk associated with pioglitazone therapy in patients with diabetes. Sci Rep. 2017;7(1):15804.

22. Beltran H, Prandi D, Mosquera JM, et al. Divergent clonal evolution of castration-resistant neuroendocrine prostate cancer. Nat Med. 2016;22(3):298-305.

23. Witkiewicz AK, McMillan EA, Balaji U, et al. Wholeexome sequencing of pancreatic cancer defines genetic diversity and therapeutic targets. Nat Commun. 2015;6:6744.

24. Ferwana M, Firwana B, Hasan R, et al. Pioglitazone and risk of bladder cancer: a meta-analysis of controlled studies. Diabet Med. 2013;30(9):1026-32.

25. Culig Z. Androgen receptor coactivators in regulation of growth and differentiation in prostate cancer. J Cell Physiol. 2016;231(2):270-4.

26. Vageli DP, Doukas SG, Sasaki CT. Inhibition of NFkappaB prevents the acidic bile-induced oncogenic mRNA phenotype, in human hypopharyngeal cells. Oncotarget. 2018;9(5):5876-91.

27. Wang L, Wu H, Wang L, et al. Asporin promotes pancreatic cancer cell invasion and migration by regulating the epithelial-to-mesenchymal transition (EMT) through both autocrine and paracrine mechanisms. Cancer Lett. 2017;398:24-36.

28. Witte D, Bartscht T, Kaufmann R, et al. TGF-beta1induced cell migration in pancreatic carcinoma cells is RAC1 and NOX4-dependent and requires RAC1 and NOX4-dependent activation of p38 MAPK. Oncol Rep. 2017;38(6):3693-701.

29. Tsubaki M, Takeda T, Tomonari Y, et al. Pioglitazone inhibits cancer cell growth through STAT3 inhibition and enhanced AIF expression via a PPARgamma-independent pathway. J Cell Physiol. 2018;233(4):3638-47.

30. Ramos-Nino ME, MacLean CD, Littenberg B. Association between cancer prevalence and use of thiazolidinediones: results from the Vermont Diabetes Information System. BMC Med. 2007;5:17.

31. Wang Z, Wang N, Liu P, Xie X. AMPK and cancer. EXS. 2016;107:203-26.

32. Orgel E, Mittelman SD. The links between insulin resistance, diabetes, and cancer. Curr Diab Rep. 2013;13(2):213-22. 